\title{
Elicitation of Vascular Diameter in Digital Retina
}

\author{
Shiny Priyadarshini J \\ Madras Christian College, Tambaram, Chennai, 600059, India \\ Gladis D \\ Presidency College, Chennai, 600005, India
}

\begin{abstract}
The Human retina has network of blood vessel with a neat, unique biometric pattern that helps in personal identification. So the biometric pattern analysis along with vascular diameter provides better accuracy results for personal identity of normal human retina as well as for human with retinal disorders. This paper focuses on the new method in the estimation of blood vessel diameter through minutiae markings in a digital fundus image. It highlights the extraction of blood vessel pattern using kirsch's template and further enhanced for noise removal and accuracy using edge detection and filters. The minutiae points are extracted to take the termination count by applying crossing number concept. The distance is finally calculated between the two extracted termination points. The result proves that the both termination count and vessel diameter is accountable for personal identification especially for humans with retinal disorders.
\end{abstract}

Keywords - Blood vessel; Kirsch template; Termination; Vascular diameter

\section{INTRODUCTION}

The human eye is a high-tech digital camera which is one of the most complex organs in a human body. The eyeball can be divided into major portions as front and rear view. The front portion includes the Iris, lens, cornea, aqueous humor, pupil and chambers while the rear portion includes retina, optic disc, macula, fovea, optic nerves and blood vessels. The typical structure of a human eyeball is shown in Fig.1(a).

The retina is a thin layer which is connected to brain through optic nerve. It converts light into neural signals which is sent to brain and is the sole reason for visual recognition. The retina is made up of network of blood vessels forming a unique biometric identity ${ }^{6}$. The structural pattern is very unique for each individual and makes it a very high secured personal identification mark. An analysis is made on the retinal blood vessel pattern to retrieve its characteristic points along with its diameter. The retinal image with its important parts is shown in Fig 1.

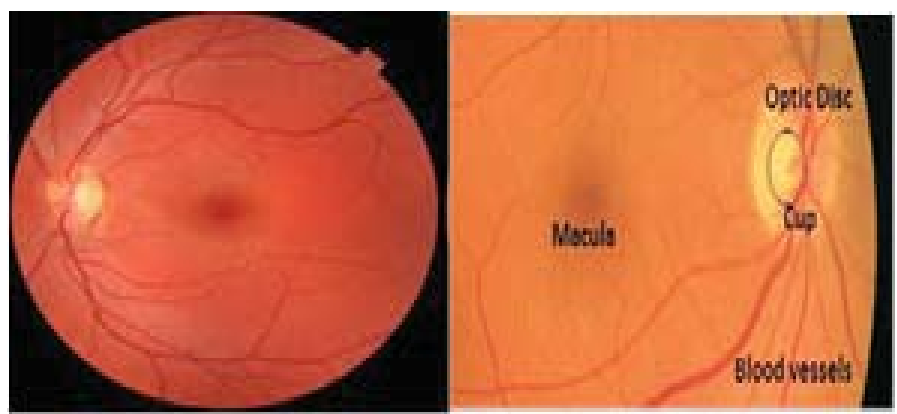

Fig.1 Sample Retina with important parts

\section{EXISTING WORK}

Conor Heneghana et.al (2002) presented the changes in blood vessel by calculating vessel thickness. Allaudin et.al (2008) presented the blood vessel diameter calculation by using various segmentation techniques like space transformation, feature extraction, gabor filter and adaptive region growing etc. Jerald et.al (2010) presented the extraction of main vessel and branching vessel by calculating the thickness of blood vessel by filtering, binarization and skeletonization techniques. M.Kalaivani et.al (2012) presented the blood vessel extraction using Kirsch's 
filt

biometric identity by calculating the Euclidean distance. H.S. Bhadauria et.al (2013) presented the Vessel extraction from retinal images using Kirsch's template. Nidhal El Abbadi et.al (2014) presented the blood vessel diameter measurement by plotting bifurcation points and later removing to find the blood vessel diameter using Euclidean distance. Xiaohong Gao et.al presented the determination of vessel diameters to study the cardio vascular diseases.

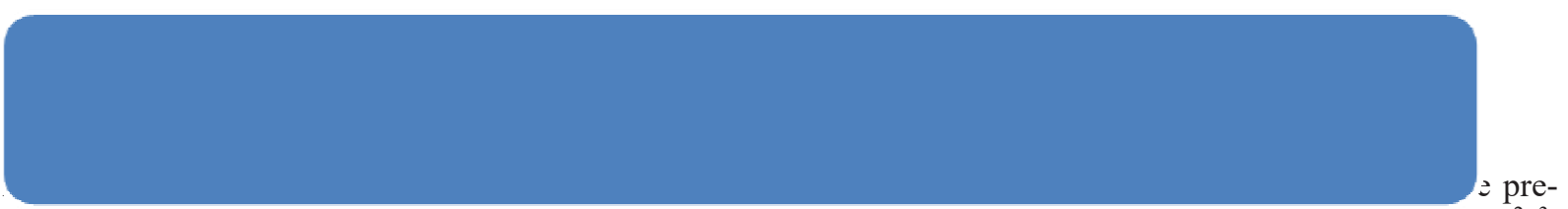
processing stage along with image enhancement techniques using Median filter and Edge detection techniques ${ }^{2,3}$. The Post-processing stage focuses on the minutiae marking using crossing number concept and the blood vessel diameter. The spurious minutiae are removed and further analyzed for calculation of blood vessel thickness using Euclidean's Distance ${ }^{8}$
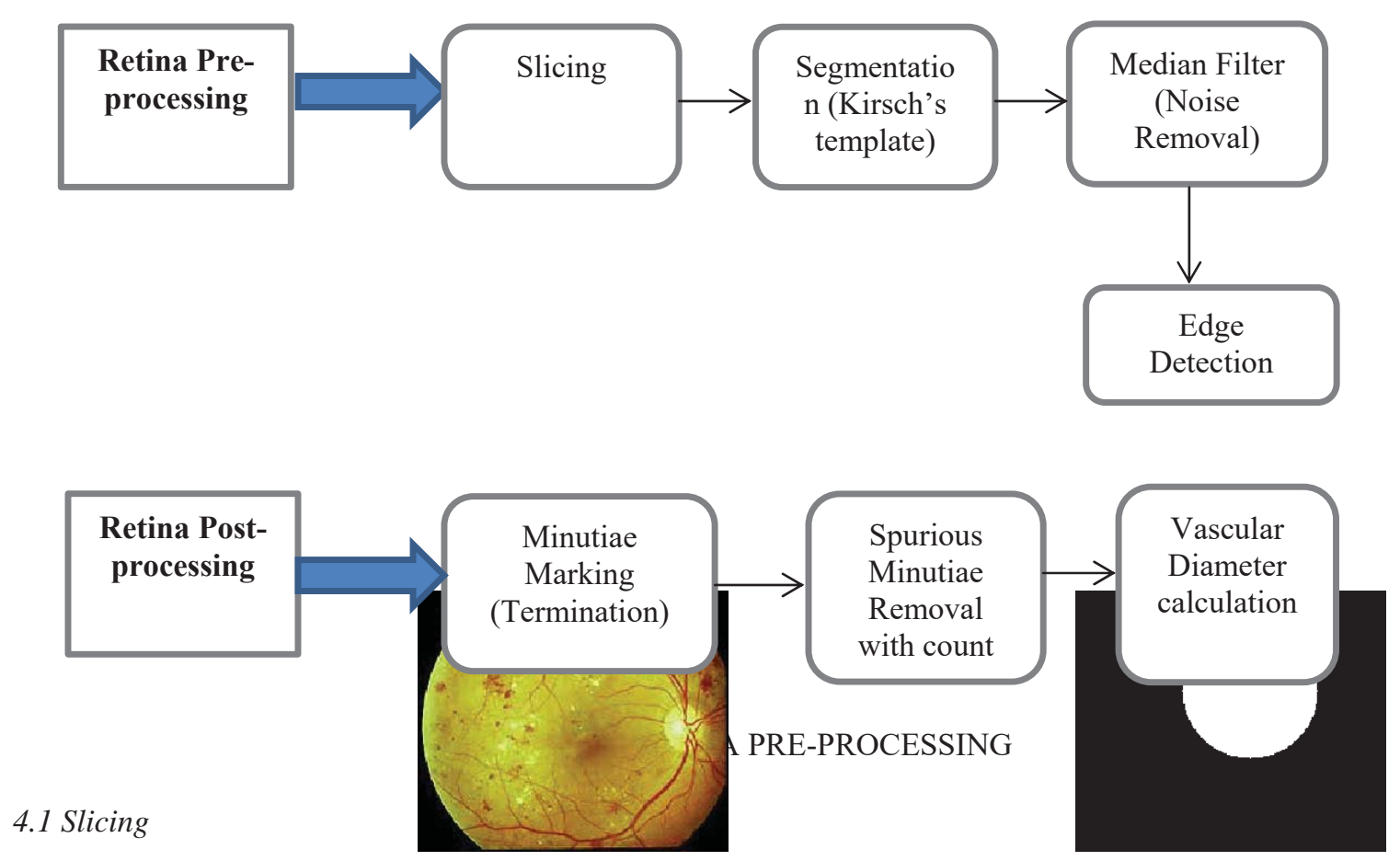

It is a process where a small portion of an image is sliced to a particular structure. Here the image is masked to a circular structure by setting its location and radius to the given input image. The image is sliced based on the radius along with its $\mathrm{X}, \mathrm{Y}$ axis which represents its given retinal image for accuracy. The original image with

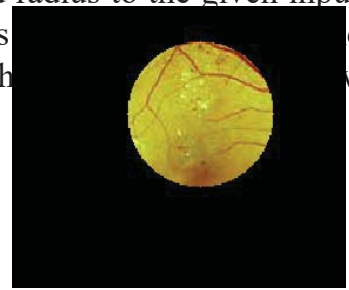
centrate on a particular region for a wn in Fig 2. 

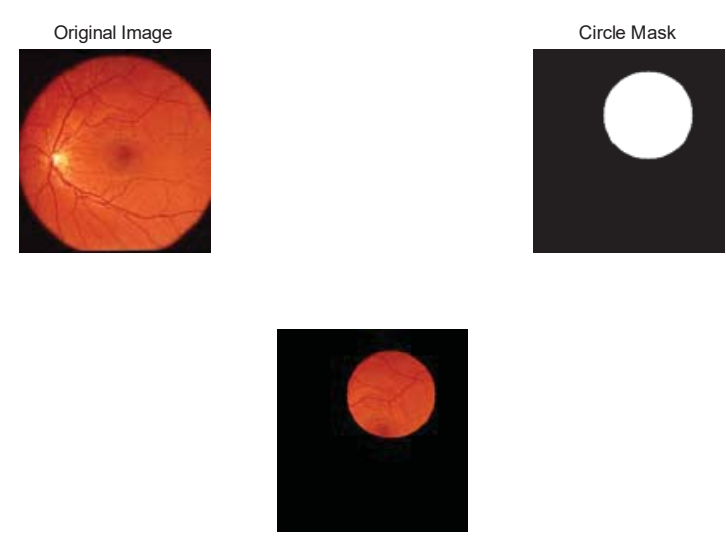

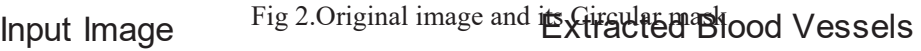

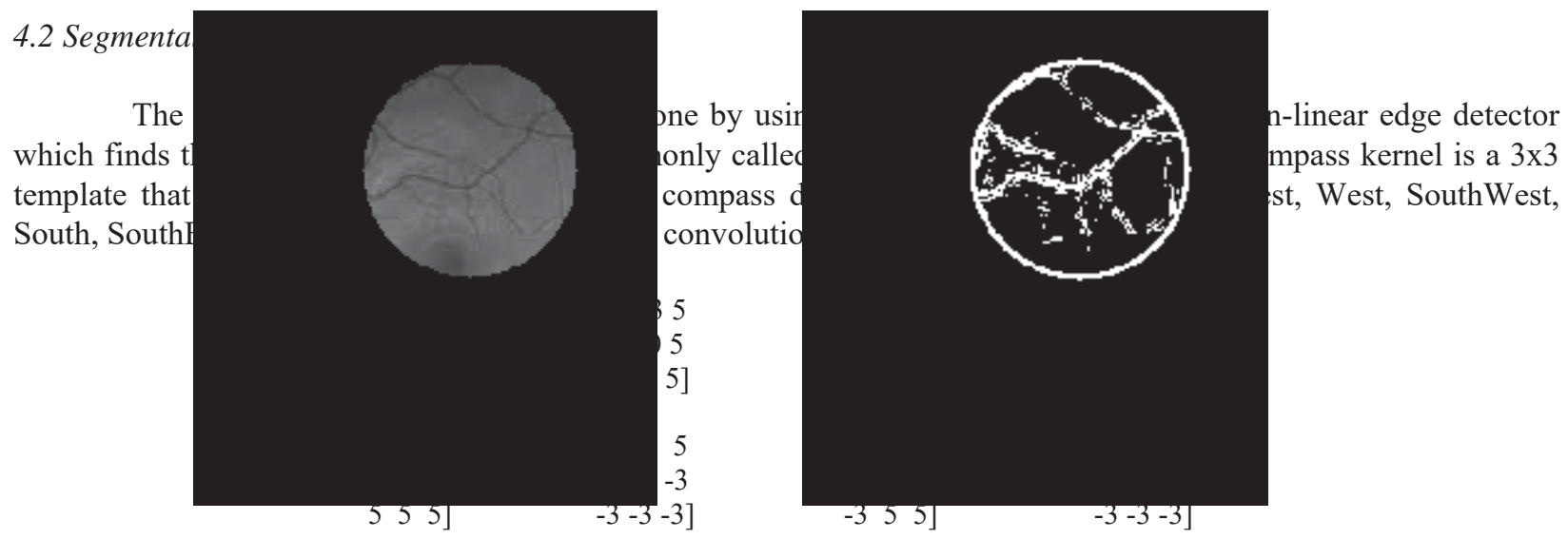

Using the above templates the image is filtered again using a two dimension filter to get the accurate edges which is shown in Fig. 3
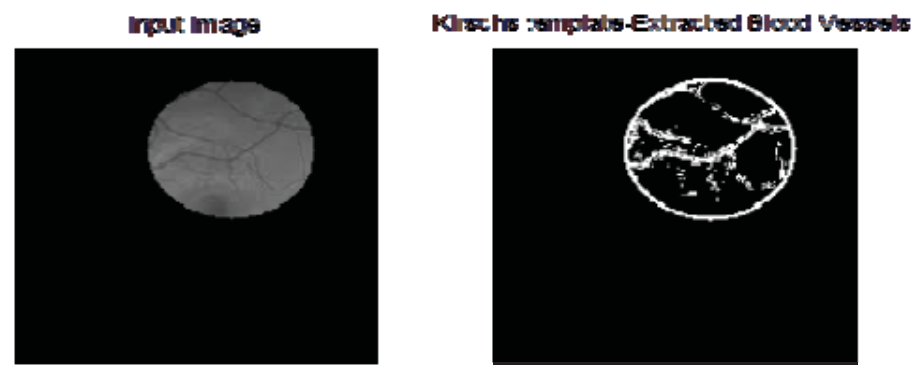

Fig 3.Original image and Kirsch's Edge detector image

4.3 Median Filter 
The median filter is applied to the extracted blood vessel. The two dimensional median filtering is used to enhance the image by reducing the noise. In each $3 \times 3$ window the median filter is padded with 0 's and removes noise by removing pixels. The median filtered image is shown in Fig.4(c) which clearly depicts the removal of noise.
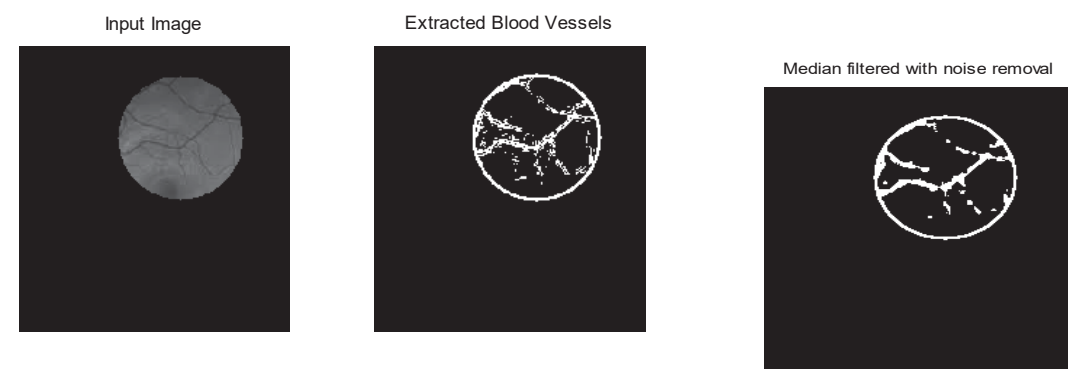

Fig 4(a).Input image; 4(b) Blood vessel extracted image; 4(c) Median filtered image

\subsection{Edge Detection}

The Canny Edge detection filter is applied to the median filtered image. The canny operator highlights the edges of an image and sets zeros to all other pixels which do not form the edges until it forms to a thin line output. The retinal image with the canny edge detection is shown in Fig 5.

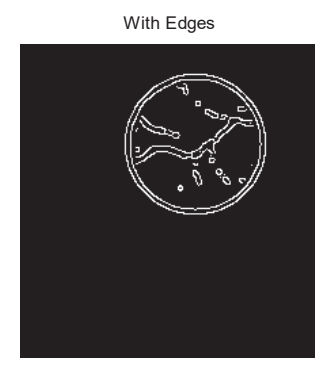

Fig 5. Canny Edge detected image

\section{RETINA POST-PROCESSING}

\subsection{Termination Marking and Spurious Removal}

The retinal pattern has tiny characteristic points called minutiae. For each $3 \times 3$ window the termination points are marked by the crossing number concept. For a termination the central pixel should be one with exactly only one valued adjacent pixel then the pixel is marked as a termination marking. Fig 6(a) depicts the image with spurious termination markings. The average inter ridge distance $\mathrm{D}$ is calculated to remove the spurious markings. If the distance between two terminations is less than 3 then the termination point is unmarked and removed. Finally a count is made on the number of terminations marked in the retina. Fig 6(b) depicts the non-spurious termination markings. 

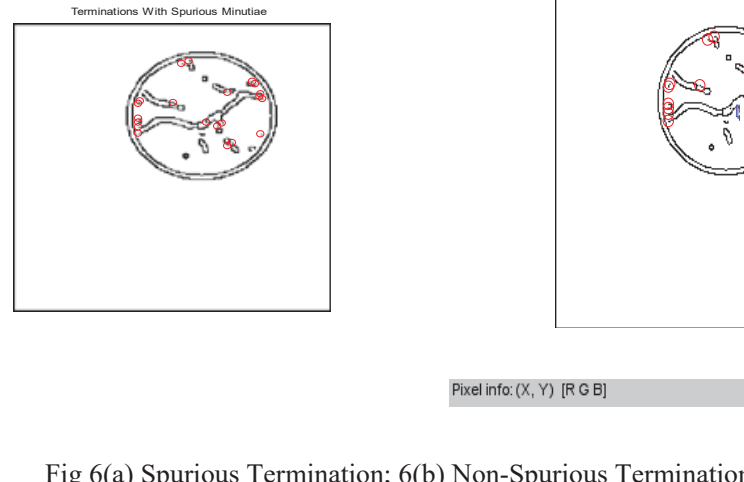

Fig 6(a) Spurious Termination; 6(b) Non-Spurious Termination

\subsection{Blood Vessel Diameter}

The thickness of the blood vessel is calculated between the two termination points in the masked retinal image $^{8}$. The distance calculated is the Euclidean's distance between the two extracted termination points. The results are depicted in pixel value which is shown in Fig.7 (a). with its enlarged images in Fig.7(b) and 7(c) respectively.
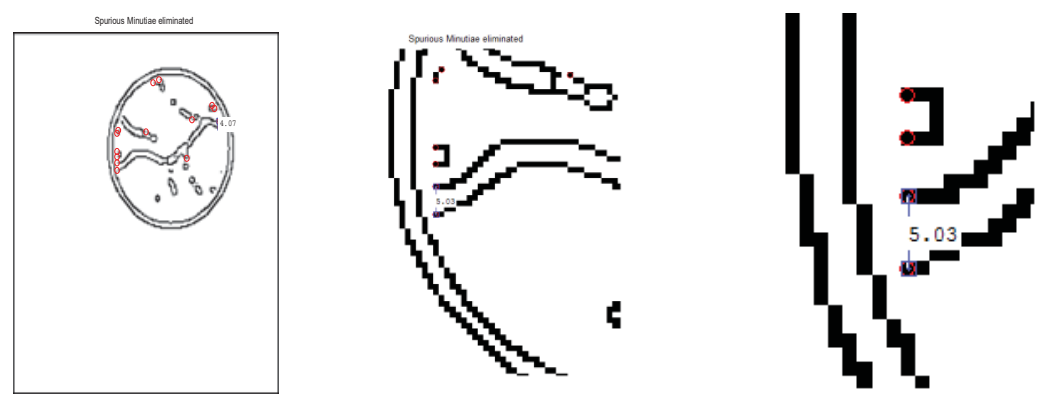

Fig 7(a) Blood vessel with thickness; 7(b), (c) Enlarged images

\section{SIMULATION RESULTS}

The vascular structure of the retinal image is segmented and enhanced in the pre-processing stage using the Kirsch' algorithm, median filter and Edge detector. In the post-processing stage the minutiae points are identified and the terminations are marked with count. The results show that the Kirsch's algorithm performs better for normal retina when compared to abnormal retina which shows less accuracy in blood vessel extraction and thus leading to increase in termination count. Hence for diseased retina when the diameter thickness is calculated it can provide a better personal identity results. The two terminations of a particular vessel is taken in the retinal image which gives the Euclidean's distance between the walls of the blood vessel. The above mentioned steps are shown clearly in Fig. 8 which represents the different stages in retrieving the distance for the five retinal images chosen randomly from the DRIVE database. The first three images are the normal retinal images and the last two images shows the diseased retina.

Table I depicts a clear picture about the vessel thickness of each retinal image along with its termination count. Here the results show that the termination count is increased for diseased retina when compared to normal retina. Table II gives detailed information of a single retinal image R3 whose diameter is calculated in two different locations. 


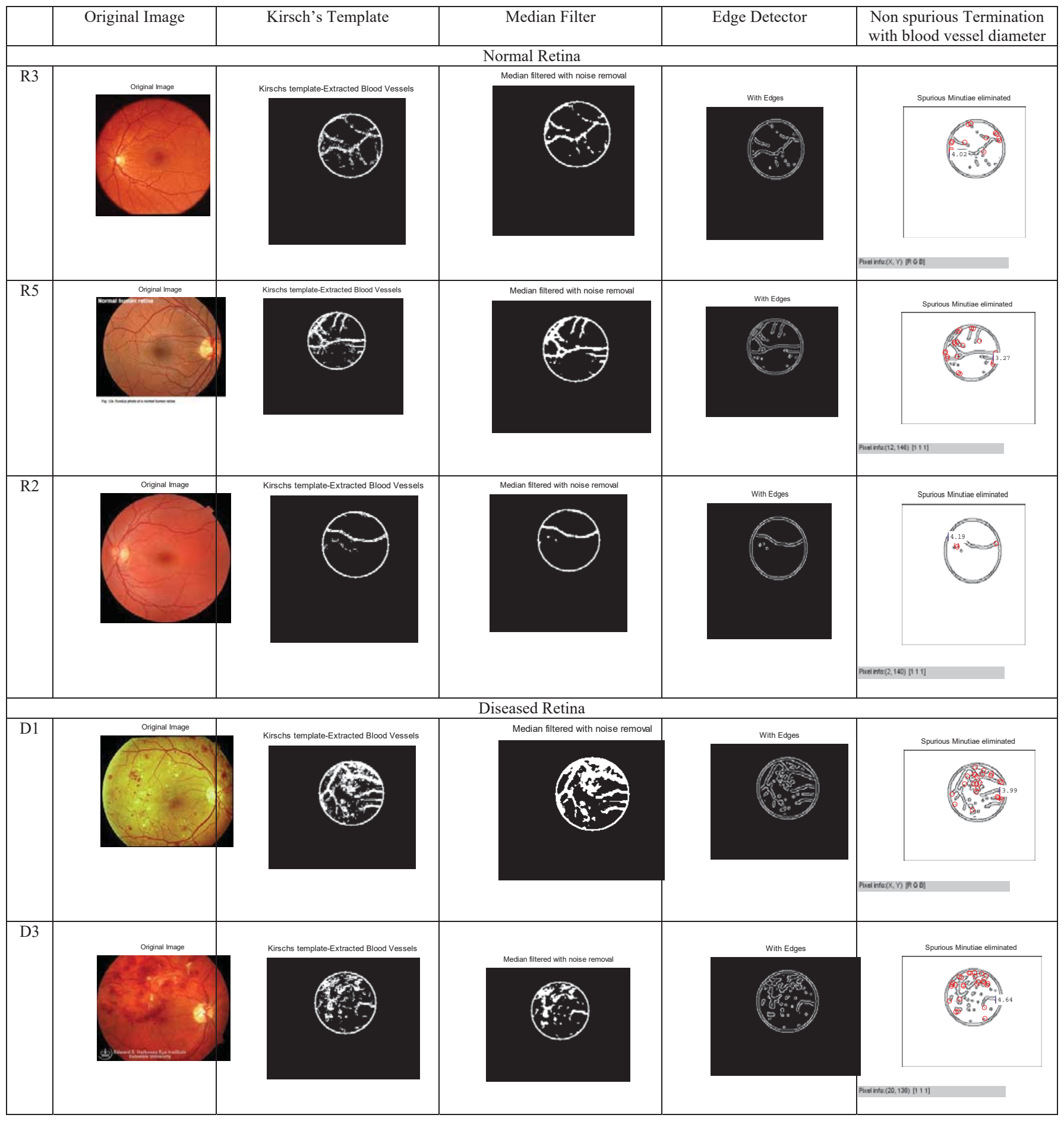

Fig. 8 
Table 1. Vascular thickness of Retina in pixels

\begin{tabular}{llll}
\hline Normal/ Diseased & Retinal Names & Blood vessel thickness in pixel & $\begin{array}{l}\text { Non-spurious Termination } \\
\text { Count }\end{array}$ \\
\hline Normal & R3 & 4.02 & $\mathbf{1 5}$ \\
Normal & R5 & 3.27 & $\mathbf{2 1}$ \\
Normal & R2 & 4.19 & $\mathbf{5}$ \\
Diseased & D1 & 3.99 & $\mathbf{2 6}$ \\
Diseased & D3 & 4.64 & $\mathbf{2 7}$ \\
\hline
\end{tabular}

Table 2. Vascular thickness of Retina 3 in two different locations

\begin{tabular}{lll}
\hline Retina- R3 & Diameter=4.92 & Diameter=4.18 \\
\hline Spurious Minutiae eliminated & Spurious Minutiae eliminated & Spurious Minutiae eliminated \\
Pixel inf: $(X, Y)[R G$ B] & Pixel inf:(153,66) [111]
\end{tabular}

\section{CONCLUSION}

It can be concluded that the Personal recognition of an individual can be strengthened by calculating blood vessel diameter in addition to minutiae markings. Because the results show that the termination count gets increased for diseased retina which shows only moderate accuracy for personal identification hence the termination count along with blood vessel thickness is taken. The results can be further extended for medical analysis to examine the presence of retinal disorders. Thus the results prove that the vascular diameter along with termination points provides better personal identification recognition predominantly for humans with retinal anomalies.

\section{REFERENCES}

[1] Conor Heneghana, John Flynna, Michael O'Keefe, Mark Cahill, Characterization of changes in blood vessel width and tortuosity in

[2] retinopathy of prematurity using image analysis; Medical Image Analysis, ELSEVIER, 2002

[3] Alauddin Bhuiyan, Baikunth Nath, Joselito Chua and Kotagiri Ramamohanarao. An Efficient Method For Vessel Width Measurement On

[4] Color Retinal Images, Research Gate, 2008

[5] Jerald S., Madheswaran M., Automated Thickness Measurement of Retinal Blood Vessels for Implementation of Clinical Decision Support Systems in Diagnostic Diabetic Retinopathy, International journal of Medical, Health, Biomedical, Pharmaceutical Engineering;Vol 4; 2010

[6] M. Kalaivani, M. S. Jeyalakshmi, Aparna.V, Extraction Of Retinal Blood Vessels Using Curvelet Transform And Kirsch's Templates; JETAE;

[7] ISSN 2250-2459,Nov 2012

[8] M.M. Fraz et.al, Blood vessel segmentation methodologies in retinal images- A survey, computer methods and programs in biomedicine,

[9] ELSEVIER, 2012

[10] Sunita Aeri and Sukhvinder Kaur, Vein Patterns as Bio-Metric Identifier using Euclidean Distance, International Journal of Computer

[11] Applications (0975 - 8887); March 2014

[12] H.S. Bhadauria et.al, Vessels Extraction from Retinal Images, IOSR, ISSN: 2278-8735; June 2013

[13] Nidhal El Abbadi, Enas El Saadi., Blood vessel Diameter Measurement on Retinal Image; Journal of Computer Science; ISSN: 1549-3636; 2014

[14] Xiaohong Gao. Et.al, Measurement of Vessel Diameters on Retinal Images for Cardiovascular Studies, School of Computing Science 